\title{
Autophagy counteracts apoptosis in human multiple myeloma cells exposed to oridonin in vitro via regulating intracellular ROS and SIRT1
}

\author{
Rong ZENG\#, Yan CHEN", Shuai ZHAO, Guo-hui CUI* \\ Department of Hematology, Union Hospital, Tongji Medical College, Huazhong University of Science and Technology, Wuhan 430022, \\ China
}

\begin{abstract}
Aim: To explore the mechanisms underlying the oridonin-induced apoptosis and autophagy in human multiple myeloma cells in vitro. Methods: Human multiple myeloma RPMI8266 cells were used. The cell viability was assessed using MTT assay. Morphological changes of apoptosis and autophagy were observed under transmission electron microscope. TUNEL and annexin V-FITC/PI dual staining assays were used to measure apoptosis. Autophagy was analyzed using Western blot analysis and immunofluorescence staining with a QDs $605 \mathrm{~nm}$-Anti-LC3 fluorescent probe. Intracellular ROS was estimated with flow cytometry using DCFH-DA fluorescent probe. Protein levels of active caspase 3, Beclin 1 and SIRT1 were determined with Western blot analysis.

Results: Exposure to oridonin (1-64 $\mu \mathrm{mol} / \mathrm{L})$ inhibited the proliferation of RPMI8266 cells in a concentration-dependent manner with an $\mathrm{IC}_{50}$ value of $6.74 \mu \mathrm{mol} / \mathrm{L}$. Exposure to oridonin $(7 \mu \mathrm{mol} / \mathrm{L})$ simultaneously induced caspase 3-mediated apoptosis and Beclin 1-dependent autophagy of RPMI8266 cells. Both the apoptosis and autophagy were time-dependent, and apoptosis was the main effector pathway of cell death. Exposure to oridonin $(7 \mu \mathrm{mol} / \mathrm{L})$ increased intracellular ROS and reduced SIRT1 nuclear protein in a time-dependent manner. The blockade of intracellular generation of ROS by NAC (5 mmol/L) abrogated apoptosis, autophagy and the decrease of SIRT1 in the cells exposed to oridonin $(7 \mu \mathrm{mol} / \mathrm{L})$. The inhibition of autophagy by 3-MA (5 mmol/L) sensitized the cells to oridonin-induced apoptosis, which was accompanied by increased intracellular ROS and decreased SIRT1.

Conclusion: Oridonin simultaneously induces apoptosis and autophagy of human multiple myeloma RPMI8266 cells via regulation of intracellular ROS generation and SIRT1 nuclear protein. The cytotoxicity of oridonin is mainly mediated through the apoptotic pathway, whereas the autophagy protects the cells from apoptosis.
\end{abstract}

Keywords: oridonin; autophagy; apoptosis; ROS; SIRT1; human multiple myeloma cells

Acta Pharmacologica Sinica (2012) 33: 91-100; doi: 10.1038/aps.2011.143; published online 12 Dec 2011

\section{Introduction}

Oridonin, an active diterpenoid compound isolated from Rabdosia rubescens, has various pharmacological and physiological effects (eg, anti-tumor, anti-inflammation, and anti-bacterial), and it has been widely used for the treatment of various human diseases ${ }^{[1-3]}$. Both apoptosis and autophagy are essential cellular homeostatic mechanisms, which are important in multicellular organisms for development, tissue turnover, and host defense. Autophagy is a conserved, genetically controlled process that leads to the degradation of cytoplasmic components within lysosomes and has recently gained much attention for its paradoxical relationship with apoptosis. Some

\footnotetext{
\# The first two authors contributed equally to this work.

* To whom correspondence should be addressed.

E-mail ghcui@medmail.com.cn

Received 2011-05-08 Accepted 2011-09-23
}

studies suggest that the inhibition of autophagy enhances apoptosis. In contrast, others have suggested that autophagy acts as a cell death pathway, termed programmed cell death (PCD) type II, in cooperation with apoptosis ${ }^{[4-7]}$. Although oridonin has been shown to induce apoptosis and autophagy in some types of tumor cells in both in vitro and in vivo studies $^{[8-10]}$, the relationship between the two processes is unclear. Furthermore, the molecular mechanisms underlying oridonininduced apoptosis and autophagy in RPMI8266 cells remain to be determined.

Multiple myeloma (MM) is an untreatable hematological disease characterized by the synthesis of excess immunoglobulin (Ig), which forms endoplasmic reticulum-localized unfolded or misfolded proteins that are potentially toxic to MM cells. Hoang et al ${ }^{[11]}$ found that the level of autophagy that occurs in MM cells was significantly higher than that in normal plasma cells. Additionally, the inhibition of autophagy 
in MM cells using chloroquine or Beclin 1-siRNA leads to cell death. This observation suggests that autophagy has a protective effect on MM cell viability. Furthermore, the proteasome inhibitor bortezomib, a clinical drug used for the treatment of myeloma, can induce MM cell death and autophagy. However, the treatment of MM cells with an autophagy inhibitor in combination with bortezomib resulted in an antagonistic response in vitro ${ }^{[11]}$. Consequently, the relationship between autophagy and cell death remains complicated and requires further investigation in multiple myeloma.

Reactive oxygen species (ROS) are generally small, shortlived and highly reactive molecules. While ROS generation is a consequence of basal cellular respiration, increased ROS generation is associated with several pathological conditions (eg, hypoxia, ischemia, and anti-tumor agents). Some studies have demonstrated that ROS generation activated caspase cascades through the mitochondrial permeability transition to mediate apoptosis ${ }^{[12,13]}$. In addition to apoptosis, ROS generation has recently been reported to mediate autophagy under certain conditions ${ }^{[14-16]}$. Sirtuin1 (SIRT1) is a NAD ${ }^{+}$-dependent deacetylase that is involved in a diverse set of physiological functions, including gene silencing, stress resistance, apoptosis, inflammation, senescence, and aging. Studies have indicated that SIRT1 activity can be positively or negatively regulated by intracellular oxidative stress ${ }^{[17-19]}$. SIRT1 was suggested to have anti-apoptotic functions by a wide range of in vivo and in vitro studies ${ }^{[20,21]}$. However, SIRT1 was shown to promote autophagy ${ }^{[22,23]}$. Therefore, we hypothesized that the generation of intracellular ROS and SIRT1 activity could underlie the effects of oridonin treatment in RPMI8226 cells.

\section{Methods and materials}

\section{Reagents and antibodies}

Oridonin, thiazolyl blue tetrazolium bromide (MTT), the $2^{\prime}, 7^{\prime}$-dichlorofluorescein diacetate (DCFH-DA) fluorescent probe, dimethyl sulfoxide (DMSO), the 3-methyladenine (3-MA) autophagy inhibitor and the $\mathrm{N}$-acetylcysteine (NAC) free radical scavenger were purchased from Sigma-Aldrich. The purity of oridonin was confirmed by HPLC to be greater than $99 \%$. Oridonin was dissolved in DMSO to make a stock solution. The DMSO concentration was kept maintained $0.1 \%$ in all cell cultures, and it did not exert any detectable effect on cell growth or cell death. Anti-active caspase 3 (Abcam, ab2302) and anti-LC3 (Abcam, ab48394) were purchased from Abcam. Anti-Beclin 1 (sc11427) and anti-SIRT1 (sc74504) were purchased from Santa Cruz Biotechnology.

\section{Cell culture and treatments}

Human multiple myeloma RPMI8226 cells were purchased from American Type Culture Collection (ATCC). The cells were maintained in RPMI-1640 medium (GIBCO, 31800-022) supplemented with $10 \%$ fetal bovine serum (FBS) (TBD Biotechnology Development, TBD0022HLY) without antibiotics at $37^{\circ} \mathrm{C}$ in a $5 \% \mathrm{CO}_{2}$ humidified atmosphere. After the cells reached a steady-state of exponential growth in normal media, they were exposed to oridonin for $0,6,12$, or $24 \mathrm{~h}$ prior to the analysis. To inhibit intracellular ROS generation and autophagy, cells were pre-incubated with NAC or 3-MA, respectively, at a concentration of $5 \mathrm{mmol} / \mathrm{L}$ for $1 \mathrm{~h}$ prior to oridonin treatment.

\section{MTT assay}

A $100-\mu \mathrm{L}$ suspension of RPMI8226 cells were seeded on 96-well plates with or without oridonin at various concentrations $(1,2,4,8,16,32$, and $64 \mu \mathrm{mol} / \mathrm{L})$ at a density of $1 \times 10^{5}$ cells per well. After incubation for a designated period of time, MTT was added to each well at a final concentration of $0.5 \mathrm{mg} / \mathrm{mL}$ for $3 \mathrm{~h}$, and the resulting formazan crystals were dissolved in DMSO. Optical density was measured at $490 \mathrm{~nm}$ with background subtraction at $630 \mathrm{~nm}$ using a plate microreader (TECAN SPETRA). The growth inhibitory ratio was calculated as follows:

$$
\begin{aligned}
& \text { Growth inhibitory ratio }(\%) \\
&=\left[\left(A_{\text {control }}-A_{\text {blank of control }}\right)-\left(A_{\text {sample }}-A_{\text {blank of sample }}\right)\right] / \\
&\left(A_{\text {control }}-A_{\text {blank of control }}\right) \times 100 \%
\end{aligned}
$$

\section{Transmission electron microscopy (TEM) analysis}

After treatment, cell pellets were fixed with $2.5 \%$ glutaraldehyde in $0.1 \mathrm{~mol} / \mathrm{L}$ cacodylate buffer, $\mathrm{pH} 7.4$ at $4^{\circ} \mathrm{C}$ for at least $30 \mathrm{~min}$. After fixation, the specimens were thoroughly washed in $0.1 \mathrm{~mol} / \mathrm{L}$ cacodylate buffer and then fixed with $1 \%$ osmium tetroxide in the same buffer at room temperature (RT) for $1 \mathrm{~h}$. The specimens were dehydrated in a graded series of ethanol and then embedded in Epon. Thin sections $(0.1 \mu \mathrm{m})$ were cut, stained with uranyl acetate/lead citrate and viewed using a Hitachi H-300 TEM.

\section{Analysis of apoptosis using the TUNEL assay and FCM of AV/PI dual staining}

In this study, several approaches were used to detect apoptosis quantitatively and qualitatively, including (I) the terminal deoxynucleotidyl transferase mediated X-dUTP nick end labeling (TUNEL) assay and (II) annexin V-FITC (fluorescein isothiocyanate)/PI (propidium iodide) staining for FCM. The TUNEL assay was performed using a commercial kit (BOSTER Biological Technology, MK1020) according to the manufacturer's protocol. Briefly, $1 \times 10^{6}$ cells $/ \mathrm{mL}$ that were treated with 7 $\mu \mathrm{mol} / \mathrm{L}$ oridonin for $0 \mathrm{~h}$ or $24 \mathrm{~h}$ were collected and fixed in $4 \%$ paraformaldehyde at $4^{\circ} \mathrm{C}$. The fixed cells were then incubated with the TUNEL reaction mixture for $1 \mathrm{~h}$ at $37^{\circ} \mathrm{C}$, followed by the addition of a peroxidase-conjugated detection antibody. DNA fragments were stained using diaminobenzidine (DAB) as a substrate for the peroxidase. Positive staining was identified using a light microscope as brown granules. The apoptosis rate was calculated as follows: apoptotic rate $(\%)=$ number of positively stained cells/total number of cells $\times 100 \%$ (at least 500 cells were counted under a light microscope). For annexin V-FITC/PI dual staining, cells were processed with an Annexin V-FITC kit (Keygene, KGA108) following treatment according to the manufacturer's instructions. Next, the samples were analyzed using the FACScan flow cytometer 
(Becton Dickinson) to quantify the apoptotic rate. Different subpopulations were distinguished using the following criteria: Q1, annexin V-negative, but PI-positive (ie, necrotic cells) indicating autophagic cell death in this study; Q2, annexin V/ PI-double positive (ie, late apoptotic cells); Q3, annexin V/ PI-double negative (ie, live cells); Q4, annexin V-positive, but PI-negative (ie, early apoptotic cells). The apoptotic rate was determined as the percentage of $\mathrm{Q} 2+\mathrm{Q} 4$.

Immunofluorescence analysis of LC3 localization using a QDs $_{605} \mathrm{~nm}$ (quantum dots $605 \mathrm{~nm}$ )-Anti-LC3 fluorescent probe

To prepare the $\mathrm{QDs}_{605} \mathrm{~mm}$-Anti-LC3 fluorescent probe, coreshell QDs (ZnS-capped CdSe) were synthesized by the College of Chemistry and Molecular Sciences, Wuhan University. We used a 1.5-mL solution of high-quality oil-soluble coreshell $\mathrm{QD}_{605 \mathrm{~nm}}$ to synthesize water-soluble QDs according to a previously developed procedure ${ }^{[24]}$. These activated dots modified with thioglycolic acid were dissolved in PBS $(0.08$ mol/L, pH 7.4) containing 50 mmol EDC (1-(3-dimethyl-aminopropyl)-3-ethylcarbodiimide hydrochloride, $>98 \%)$ and 5 mmol NHS (N-hydroxy-succinimide, $>98 \%$ ). Next, the QDs were incubated with $20 \mu \mathrm{L}$ of a mouse anti-human monoclonal LC3 antibody at RT in a shaking incubator for $2-4 \mathrm{~h}$. The final QD bioconjugates were purified by centrifugation at $6000 \times g$ for $10 \mathrm{~min}$, and the suspension was dialyzed for $8-12 \mathrm{~h}$. The resulting $\mathrm{QD}_{605} \mathrm{~nm}-\mathrm{Anti}-\mathrm{LC} 3$ probes were stored at $4{ }^{\circ} \mathrm{C}$. For the immunofluorescence analysis, cells were collected following treatment and fixed in $4 \%$ paraformaldehyde for $1 \mathrm{~h}$ at $4{ }^{\circ} \mathrm{C}$. Next, the fixed cells were immobilized on a gelatin-covered $(0.1 \%$ gelatin and $0.01 \%$ chromium potassium sulfate) slide and dried under sterile conditions at RT for $1 \mathrm{~h}$. The specimen was permeabilized in phosphate buffer solution (PBS) containing $0.1 \%$ Triton X-100 and sodium citrate at RT for $10 \mathrm{~min}$. Then, the specimen was incubated with the $\mathrm{QD}_{605 \mathrm{~nm}}$-Anti-LC3 probes at a final concentration of $1 \times 10^{7} \mathrm{~mol} / \mathrm{L}$ at RT for $4 \mathrm{~h}$. After incubation, the slides were washed three times with PBS and observed using fluorescence microscopy (Olympus) with an excitation wavelength of $605 \mathrm{~nm}$ to determine LC3 localization. Under normal conditions, LC3-II is uniformly distributed and has a diffuse localization pattern. During autophagy, LC3-I is processed to LC3-II and translocates to autophagosome membranes, which appear as red fluorescent punctae. Because LC3-II localization is used as a marker for autophagy, the percentage of fluorescent punctae-positive cells compared with the total number of cells was calculated as follows to quantify the amount of autophagy: the percentage of fluorescent punctae-positive cells $(\%)=($ the number of fluorescent punctae-positive cells/the total number of cells) $\times 100 \%$ (at least 500 cells were counted using a fluorescence microscope).

\section{Measurement of intracellular ROS generation}

Intracellular ROS generation was estimated by FCM using the DCFH-DA fluorescent probe. Briefly, the treated cells were collected and washed twice with RPMI-1640. The level of intracellular ROS was determined by incubating the cells with the DCFH-DA working solution $(25 \mu \mathrm{mol} / \mathrm{L})$ at $37^{\circ} \mathrm{C}$ for 30 min. After incubation, cells were washed twice with RPMI1640 and then analyzed using FCM to determine the DCF fluorescence intensity at excitation and emission wavelengths of $488 \mathrm{~nm}$ and $525 \mathrm{~nm}$, respectively.

\section{Protein extraction and Western blot analysis}

Total cellular protein was harvested by washing cells with ice-cold PBS and incubating them in lysis buffer $(10 \mathrm{mmol} / \mathrm{L}$ Tris, $\mathrm{pH}$ 7.4, $20 \mathrm{mmol} / \mathrm{L} \mathrm{NaCl}, 5 \mathrm{mmol} / \mathrm{L} \mathrm{MgCl}_{2}, 0.5 \% \mathrm{NP}-40$, and $0.1 \mathrm{mmol} / \mathrm{L}$ PMSF). The extracts were centrifuged, and the clear supernatants containing total protein were collected. Cellular nucleic proteins were extracted using a commercial kit (DBI Bioscience, DBI1017) according to the manufacturer's protocol. After isolation, the protein concentration was determined using the Bio-Rad protein assay, and an equal amount of protein was subjected to SDS-polyacrylamide gel electrophoresis and transferred to nitrocellulose membranes (Hybond-C extra, GE Healthcare Life Sciences, RPN203E). After blocking with 5\% non-fat milk, the membranes were probed with the designated primary and secondary antibodies, developed with the enhanced chemiluminescence method (Pierce, 32106) and visualized using the Kodak Image Station. The band density was quantified using the Image J image processing program. Because the extent of LC3 conversion is correlated with the level of autophagy ${ }^{[25]}$, LC3-I and LC3-II were detected by Western blot analysis.

\section{Statistical analysis}

All data are presented as mean \pm standard deviation (SD). Statistical significance was determined using Student's $t$-test with $P$-values $<0.05$ representing significance.

\section{Results}

\section{Oridonin inhibits the proliferation of RPMI8226 cells}

RPMI8226 cells were treated with oridonin at various concentrations $(1,2,4,8,16,32$, and $64 \mu \mathrm{mol} / \mathrm{L})$ for 6,12 , or $24 \mathrm{~h}$. As shown in Figure 1, oridonin induces remarkable inhibition of cell proliferation in a time- and dose-dependent manner with an $\mathrm{IC}_{50}$ of $6.74 \mu \mathrm{mol} / \mathrm{L}$ in RPMI8226 cells at $24 \mathrm{~h}$.

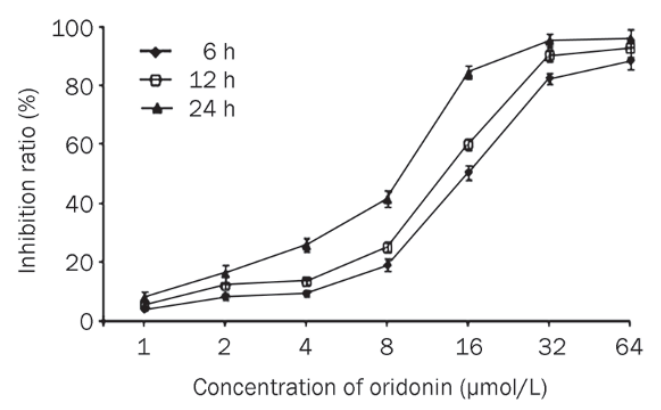

Figure 1. Assessment of the proliferation inhibition induced by oridonin in RPMI8226 cells. The cells were treated with oridonin at various doses for 6,12 , and $24 \mathrm{~h}$. Viable cells were detected by MTT assay and proliferation inhibitory ratio (\%) was calculated. The data were represented as mean $\pm S D$ from three independent experiments. 
Oridonin induces caspase 3-mediated apoptosis in RPMI8226 cells

The TUNEL assay, FCM analysis of annexin V-FITC/PI dual staining and TEM were performed to detect apoptosis in cells treated with $7 \mu \mathrm{mol} / \mathrm{L}$ oridonin for $0,6,12$, or $24 \mathrm{~h}$. As shown in Figure 2A, a significant increase in the number of TUNELpositive apoptotic cells was detected in cells treated with oridonin for $24 \mathrm{~h}$. The apoptotic rate of cells exposed to oridonin was increased to $41.4 \% \pm 1.42 \%$ while the apoptotic rate of the control group was only $5.78 \% \pm 0.56 \%$. Annexin V-FITC/PI dual staining demonstrated that oridonin induces a significant time-dependent increase in the apoptotic rate [(Q2+Q4)\%]. As shown in Figure 2C, the apoptotic rates of cells at the indicated time points were $5.00 \% \pm 1.55 \%(0 \mathrm{~h}), 10.47 \% \pm 0.68 \%(6$ h), $14.70 \% \pm 0.60 \%$ (12 h), and $17.53 \% \pm 0.68 \%$ (24 h). Apoptotic morphology was also observed using TEM in cells treated with oridonin for $24 \mathrm{~h}$ (Figure 3B).

To explore the mechanism underlying the time-dependent increase in apoptosis induced by oridonin, the level of active caspase 3 was determined using Western blot analysis of cells treated with $7 \mu \mathrm{mol} / \mathrm{L}$ oridonin for $0,6,12$, or $24 \mathrm{~h}$. As shown in Figure $2 \mathrm{~B}$, oridonin also results in a time-dependent activation of caspase 3 . These results indicate that oridonin induces caspase 3-mediated apoptosis in RPMI8226 cells.

\section{Oridonin induces Beclin 1-mediated autophagy in RPMI8226 cells}

Detection of autophagosomes, in addition to LC3 conversion and localization in cells treated with $7 \mu \mathrm{mol} / \mathrm{L}$ oridonin for 0 , 6,12 , or $24 \mathrm{~h}$ was performed using TEM, Western blot analysis, and immunofluorescence using the $\mathrm{QDs}_{605} \mathrm{~nm}$-Anti-LC3 fluorescent probe, respectively. There were a greater number of autophagosomes, which is indicative of autophagy, in cells exposed to oridonin for $24 \mathrm{~h}$ as compared with untreated controls (Figure 3C). LC3-II was detected using Western blot analysis. As shown in Figure 4A, the level of LC3-II protein increased in a time-dependent manner after treatment with oridonin. As shown in Figure 4B, oridonin induces a remarkable increase in red fluorescent punctae at 6, 12, and $24 \mathrm{~h}$, which is indicative of LC3-II localization in autophagosomes in cells. The percentage of red fluorescent punctae-positive cells increased in a time-dependent manner during treatment with oridonin for $24 \mathrm{~h}$. The percentages of red fluorescent punctae-positive cells at the indicated time points were $14.33 \% \pm 4.04 \%$ (0 h), 35.67\% $\pm 4.16 \%$ (6 h), 61.00\% $\pm 3.61 \%$ (12 h) and $91.00 \% \pm 2.65 \%(24 \mathrm{~h}$ ) (Figure $4 \mathrm{~B}$ ). These results suggest that oridonin induces autophagy in a time-dependent manner in RPMI8226 cells.

Because Beclin 1-independent autophagy has been reported, the levels of Beclin 1 in cells treated with $7 \mu \mathrm{mol} / \mathrm{L}$ oridonin for $0,6,12$, or $24 \mathrm{~h}$ were determined using Western blot analysis to investigate its role in autophagy induced by oridonin. As shown in Figure 4C, Beclin 1 expression followed the same time-dependent pattern as LC3 conversion and localization. These data indicate that autophagy induced by oridonin is canonical Beclin 1-mediated macro-autophagy.
Intracellular ROS generation mediates apoptosis and autophagy induced by oridonin through negative regulation of SIRT1 activity To investigate whether ROS and SIRT1 are involved in oridonin-induced apoptosis and autophagy, the levels of intracellular ROS and SIRT1 nuclear protein in cells treated with $7 \mu \mathrm{mol} / \mathrm{L}$ oridonin for $0,6,12$, or $24 \mathrm{~h}$ were assessed by the FCM analysis of DCF fluorescence intensity and Western blot analysis, respectively. As shown in Figure 5A, oridonininduced apoptosis and autophagy, was associated with a time-dependent increase in DCF fluorescence intensity. DCF fluorescence intensities at the indicated time points were $23154.67 \pm 1332.90$ (0 h), $28150.00 \pm 716.45$ (6 h), $32574.33 \pm 1908.46$ $(12 \mathrm{~h})$, and $44410.67 \pm 2478.12(24 \mathrm{~h})$. In contrast, oridonin treatment results in decreased nuclear SIRT1 protein in a timedependent manner (Figure 5B). These results suggest that the induction of apoptosis and autophagy by oridonin may be positively regulated by intracellular ROS generation and negatively regulated by SIRT1 activity.

NAC, a general free radical scavenger, was used to block ROS generation to further confirm the roles of intracellular ROS generation and SIRT1 activity in the induction of apoptosis and autophagy by oridonin. RPMI8226 cells were pre-incubated with $5 \mathrm{mmol} / \mathrm{L} \mathrm{NAC}$ for $1 \mathrm{~h}$ prior to exposure to $7 \mu \mathrm{mol} / \mathrm{L}$ oridonin for $24 \mathrm{~h}$, and then, the apoptosis, autophagy, and the SIRT1 activity analyses were repeated. As shown in Figures 5A and 6A, NAC completely inhibited the oridonin-induced increase in DCF fluorescence intensity $(22932.67 \pm 2715.65$ versus $44410.67 \pm 2478.12, P<0.01)$. An analysis of apoptosis and autophagy, including the FCM analysis of annexin V-FITC/PI dual staining, Western blot analysis of active caspase 3 and LC3-II, localization of LC3-II, and Western blot analysis of Beclin 1, were repeated in cells pre-incubated with NAC. While exposure to NAC alone for $24 \mathrm{~h}$ did not affect apoptosis and autophagy in cells $(P>0.05)$, NAC completely abrogated the oridonin-induced increase in apoptosis (Figures 2B, 2C, 6B, and 6C, $P<0.01$ ) and autophagy (Figures 4, 6D, 6E, and 6F, $P<0.01$ ). The SIRT1 nuclear protein level of cells pre-incubated with NAC was measured and compared with cells treated with oridonin alone. As shown in Figure 5B and Figure 6G, NAC reversed the oridonin-induced decrease in SIRT1 nuclear protein levels $(P<0.01)$.

These data suggest that SIRT1 activity was negatively regulated by intracellular ROS generation. Therefore, we conclude that intracellular ROS generation mediates the induction of apoptosis and autophagy by oridonin, possibly through the negative regulation of SIRT1 activity.

Inhibition of autophagy sensitizes RPMI8226 cells exposed to oridonin to apoptosis mediated by intracellular ROS generation and SIRT1 downregulation

We used 3-MA, a well-known inhibitor of autophagy, to explore the role of autophagy in cell death and the relationship between autophagy and apoptosis. Autophagy and apoptosis of cells pre-treated with $5 \mathrm{mmol} / \mathrm{L} \mathrm{3-MA}$ for $1 \mathrm{~h}$ and then exposed to $7 \mu \mathrm{mol} / \mathrm{L}$ oridonin for $24 \mathrm{~h}$ were analyzed using above mentioned methods. 
A

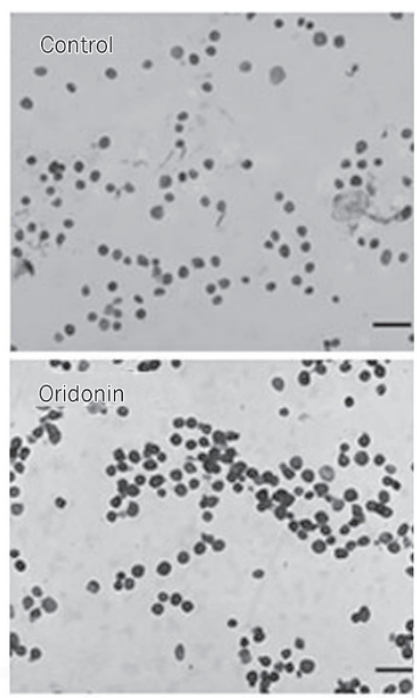

B
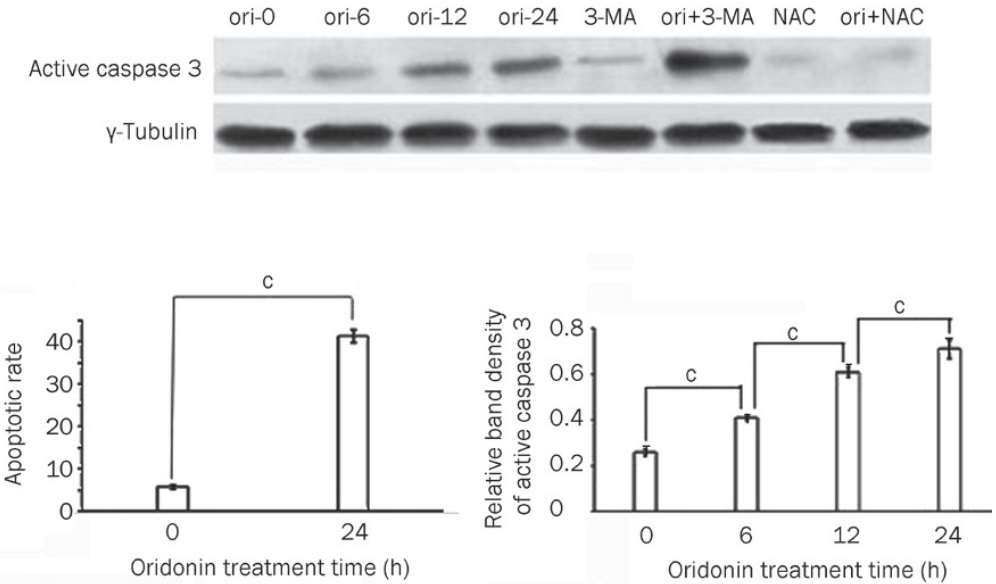

ori-6
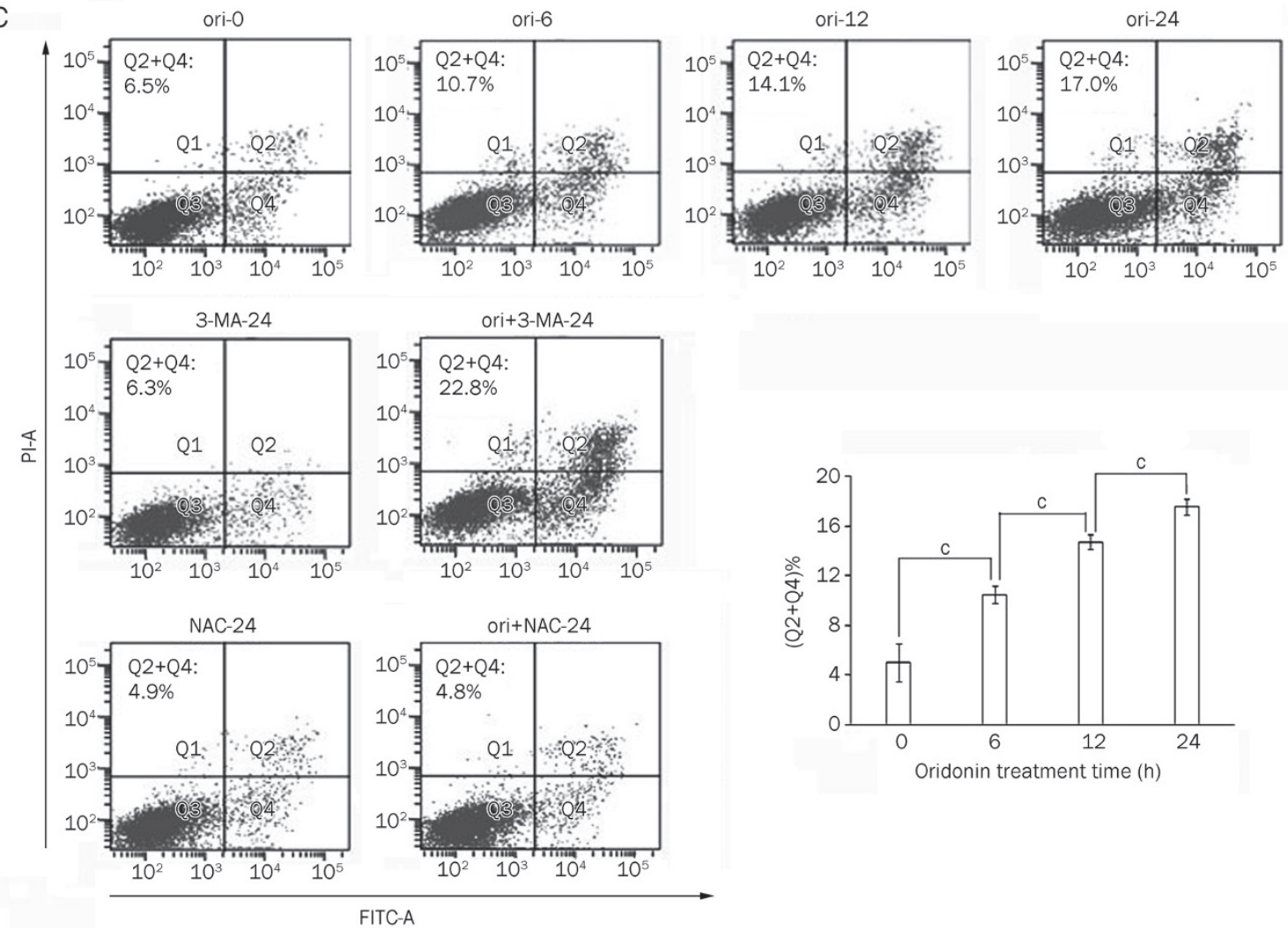

Figure 2. Assessment of apoptosis in cells treated with oridonin (ori) alone, cells pre-incubated with NAC, and cells pre-incubated with 3-MA. (A) The cells were treated with $7 \mu \mathrm{mol} / \mathrm{L}$ oridonin for $24 \mathrm{~h}$, and apoptosis was determined using TUNEL assay. The cells cultured under normal media were used as control. The positive staining was identified under the light microscope at $\times 400$ magnification as brown granules. Apoptotic rate was calculated as follows: apoptotic rate $(\%)=$ number of positive staining cells/number of total cells $\times 100 \%$ (at least 500 cells were counted under a light microscope). (B) The cells were treated with $7 \mu \mathrm{mol} / \mathrm{L}$ oridonin for $0,6,12$, and $24 \mathrm{~h}$, or treated with $7 \mu \mathrm{mol} / \mathrm{L}$ oridonin in the presence or absence of $5 \mathrm{mmol} / \mathrm{L} \mathrm{NAC}$, or treated with $7 \mu \mathrm{mol} / \mathrm{L}$ oridonin in the presence or absence of $5 \mathrm{mmol} / \mathrm{L} 3-\mathrm{MA}$ for $24 \mathrm{~h}$. The active caspase 3 protein levels were detected using Western blot assay. $y$-Tubulin was used as an equal loading control. The bands were quantified by densitometric analysis. The values for active caspase 3 were corrected relative to the $y$-tubulin and shown in histogram. (C) The cells were treated with $7 \mu \mathrm{mol} / \mathrm{L}$ oridonin for $0,6,12$, and $24 \mathrm{~h}$, or treated with 7 $\mu \mathrm{mol} / \mathrm{L}$ oridonin in the presence or absence of $5 \mathrm{mmol} / \mathrm{L} \mathrm{NAC}$, or treated with $7 \mu \mathrm{mol} / \mathrm{L}$ oridonin in the presence or absence of $5 \mathrm{mmol} / \mathrm{L} 3-\mathrm{MA}$ for $24 \mathrm{~h}$. The apoptotic rates were detected by FCM of annxin V-FITC/PI dual staining. Q1 quadrant (annexin V-, PI+) represented dead cells; Q2 quadrant (annexin $\mathrm{V}+, \mathrm{PI}+$ ) represented late apoptotic cells; Q4 quadrant (annexin $\mathrm{V}+, \mathrm{PI}-$ ) represented early apoptotic cells; Q4 quadrant (annexin V-, $\mathrm{PI}-$ ) represented live cells. The percentage of total apoptotic cells $(\mathrm{Q} 2+\mathrm{Q} 4)$ was calculated and shown in histogram. All statistical significance was determined by Student's $t$-test. Columns, mean of three independent experiments; Mean $\pm S D$; ${ }^{c} P<0.01$. 

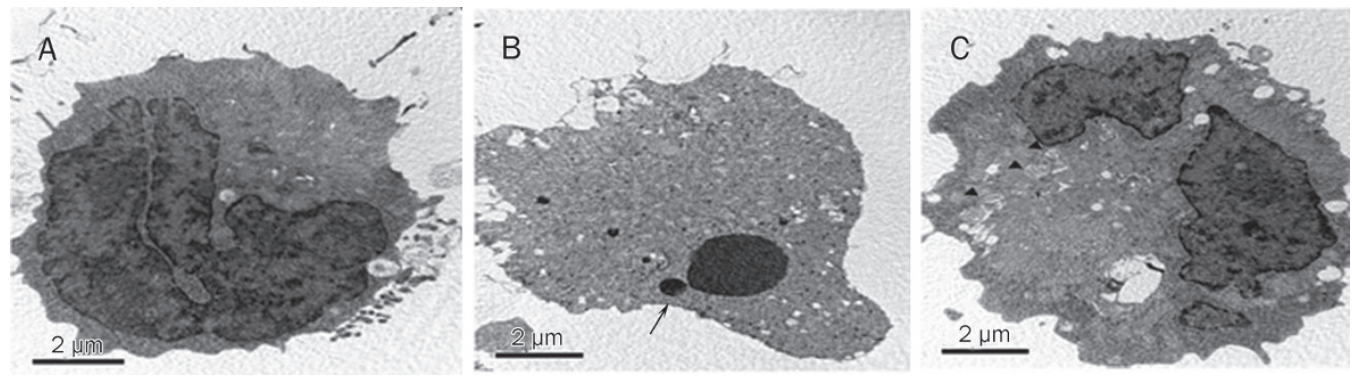

Figure 3. Assessment of the ultra-structure morphology of apoptosis and autophagy in RPMI8226 cells exposed to oridonin. The cells cultured under normal media were used as control (A). The cells were treated with $7 \mu \mathrm{mol} / \mathrm{L}$ oridonin for $24 \mathrm{~h}(\mathrm{~B}, \mathrm{C})$. The representative images are shown. The magnification was labeled in each image. (A) Normal morphology. (B) Apoptotic morphology. Arrow depicts apoptotic chromatin condensation. (C) Autophagic morphology. Arrowheads indicate autophagosomes.

3-MA suppresses oridonin-induced autophagy, but not baseline autophagy. As shown in Figures 4A, 7A, 7B, and $7 \mathrm{C}$, the amount of LC3-II, the percentage of red fluorescent punctae-positive cells and Beclin 1 protein expression in cells pre-treated with 3-MA decreased markedly compared with cells treated with oridonin alone $(P<0.01)$. In contrast, a comparison of these autophagic parameters between cells exposed to 3-MA alone and cells under normal conditions showed no significant differences $(P>0.05)$. In addition, $3-\mathrm{MA}$ enhances oridonin-induced apoptosis. As shown in Figures 2B and 7D, 3-MA augments the oridonin-induced increase in the apoptotic rate $(24.30 \% \pm 1.80 \%$ versus $17.53 \% \pm 0.68 \%, P<0.01)$. Measurement of active caspase 3 levels in cells pre-treated with 3-MA revealed the same results as the analysis of the apoptotic rate (Figure 2C and Figure 7E). In conclusion, apoptosis, but not autophagy, is the major effector pathway of oridonin-induced cytotoxicity and autophagy protects cells against apoptosis.

Because the results above demonstrated that intracellular ROS generation mediates oridonin-induced apoptosis through negatively regulating SIRT1 activity, we determined whether intracellular ROS generation and SIRT1 were also involved in the pro-survival effect of autophagy. The intracellular ROS generation and SIRT1 protein level in the nuclei of cells pretreated with $5 \mathrm{mmol} / \mathrm{L} 3-\mathrm{MA}$ for $1 \mathrm{~h}$ and then exposed to 7 $\mu \mathrm{mol} / \mathrm{L}$ oridonin for $24 \mathrm{~h}$ was detected by an FCM analysis of DCF fluorescence density and Western blot analysis, respectively. As shown in Figures 5B, 7F, and 7G, pre-treatment with 3-MA further increases DCF fluorescence intensity $(57385.55 \pm 3935.33$ versus $44410.67 \pm 2478.12, P<0.01)$ and decreases SIRT1 nuclear protein level $(P<0.01)$ compared with cells treated with oridonin alone for $24 \mathrm{~h}$.

In conclusion, we propose that intracellular ROS generation contributes to the autophagic pro-survival mechanism. Inhibition of autophagy sensitizes cells to apoptosis through promotion of ROS generation and SIRT1 downregulation induced by oridonin.

\section{Discussion}

Oridonin, a potential drug for tumor treatment, has been reported to simultaneously induce apoptosis and autophagy in some tumor cell lines, including HeLa and A431 cells, among others $^{[9,26]}$. Studies investigating the relationship between autophagy and apoptosis are complicated and depend on the cellular context and stimulus. Jia and colleagues demonstrated that the induction of autophagy was essential to TNF-a-induced apoptosis in a T-lymphoblastic leukemia cell line, which indicated that execution of apoptosis is preceded by and depends upon autophagy in this context ${ }^{[27]}$. In other cellular settings, autophagy antagonized or delayed apoptosis, and the inhibition of autophagy increased the sensitivity of the cells to apoptotic signals ${ }^{[2,28]}$. In our study, we found that oridonin could simultaneously induce caspase 3-mediated apoptosis and Beclin 1-dependent autophagy in RPMI8226 cells. The inhibition of autophagy by 3-MA sensitized the cells to apoptosis, which suggests that oridonin cytotoxicity is mainly the result of apoptosis and that autophagy acts as a pro-survival mechanism in cells exposed to oridonin.

Intracellular ROS generation plays a significant role in physiological and pathological processes, and a high level of ROS is closely associated with apoptotic cell death. Recently, many studies have reported that ROS could induce apoptosis in a variety of malignant cells ${ }^{[12,13]}$. Additionally, other studies have shown that oxidative stress could induce autophagy under certain conditions. $\mathrm{H}_{2} \mathrm{O}_{2}$ and 2-methoxyestradiol, a well-known generator of ROS, could induce autophagy, which contributed to cell death in HEK293, U87, and HeLa cells ${ }^{[29]}$. Our study demonstrated that oridonin induces a time-dependent increase in intracellular ROS generation accompanied by increases in apoptosis and autophagy. The complete inhibition of intracellular ROS generation by NAC abrogated oridonin-induced apoptosis and autophagy. These data indicate that intracellular ROS generation is required for the induction of autophagy and apoptosis by oridonin.

Hasegawa et al originally demonstrated that excess ROS positively regulates SIRT1 activity and serves as a compensatory mechanism to protect cells against apoptosis ${ }^{[18]}$. Recently, some studies have shown that SIRT1 activity was negatively regulated by intracellular ROS generation in lung epithelial cells, endothelial cells, and macrophages in response to cigarette smoke extract in vitro and in the lungs of patients with COPD (chronic obstructive pulmonary disease) ${ }^{[19,30,31]}$. In this study, we demonstrate that oridonin induces a time-dependent 
A
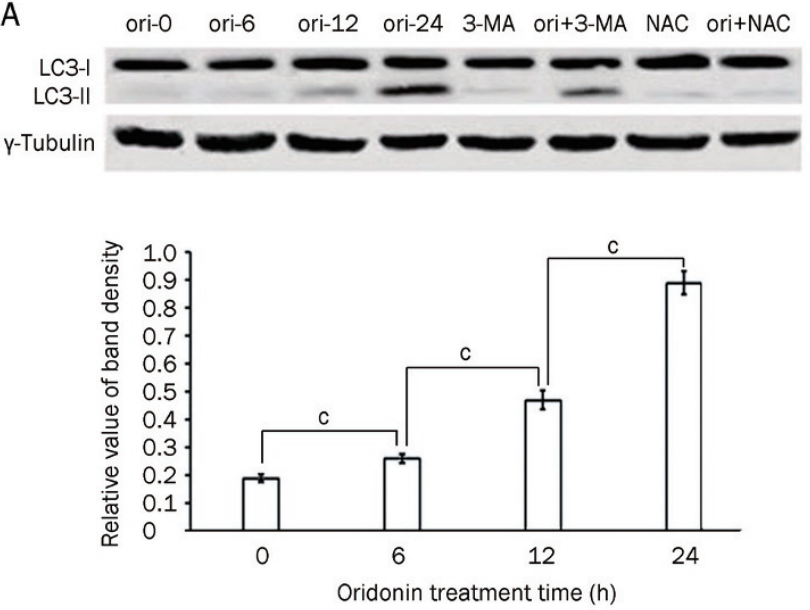

B
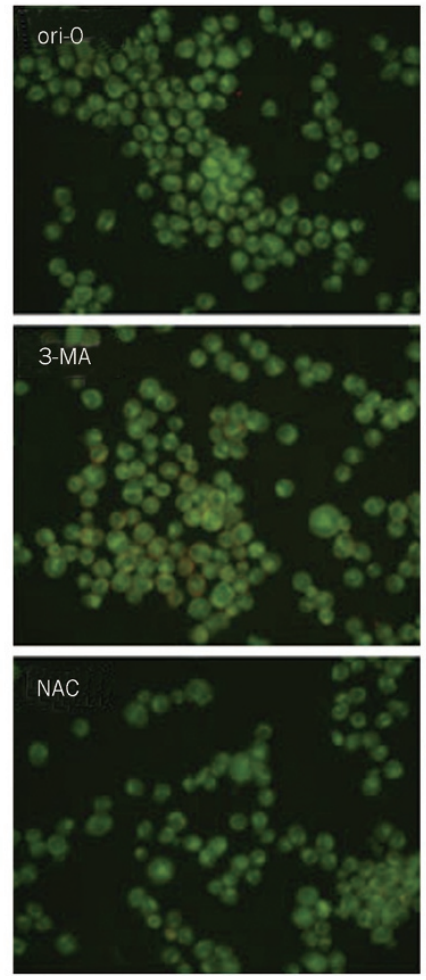

C
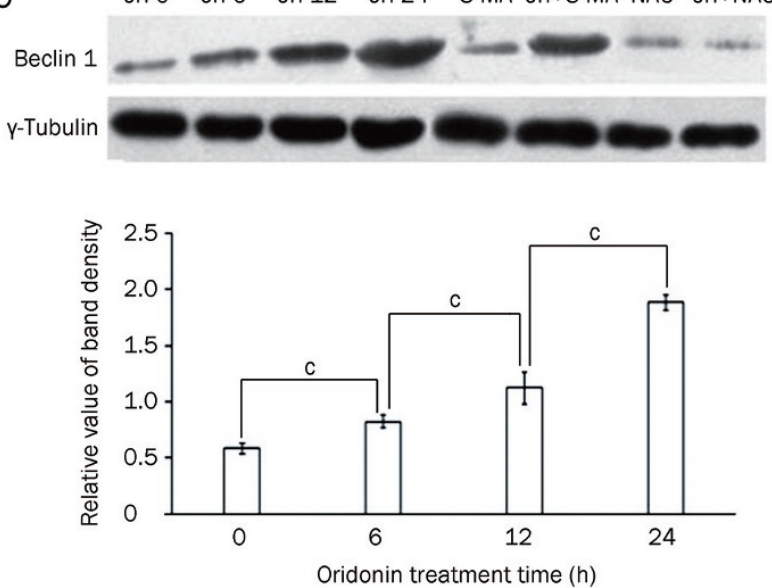

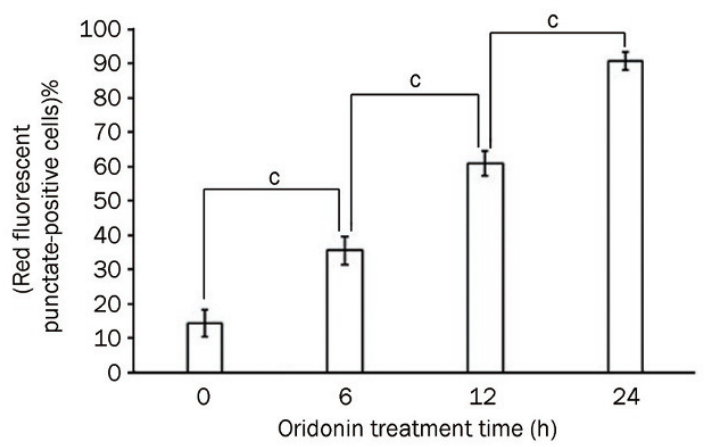

Figure 4. Autophagy of cell treated with oridonin (ori) alone, cells pre-incubated with NAC, and cells pre-incubated with 3-MA. The cells were treated with $7 \mu \mathrm{mol} / \mathrm{L}$ oridonin for $0,6,12$, and $24 \mathrm{~h}$, or treated with $7 \mu \mathrm{mol} / \mathrm{L}$ oridonin in the presence or absence of $5 \mathrm{mmol} / \mathrm{L} \mathrm{NAC}$, or treated with $7 \mu \mathrm{mol} / \mathrm{L}$ oridonin in the presence or absence of 5 mmol/L 3-MA for $24 \mathrm{~h}$. (A) The LC3-II and LC3-I protein levels of treated cells were detected using Western blot assay. $y$-Tubulin was used as an equal loading control. The bands were quantified by densitometric analysis. The LC3-II were corrected relative to $y$-tubulin. (B) After designed experiment measures, the LC3 localization in cells was determined by immunofluorcesence using a fluorescent probe of $\mathrm{QDS}_{605 \mathrm{~nm}}$-Anti-LC3, and the localization of LC3-II at autophagosome membrane were indicated as red fluorescent punctate dots. The representative images are shown. Autophagy was quantitated by the percentage of fluorescent punctate-positive cells in total cells. the percentage of fluorescent punctate-positive cells was calculated as follows: the percentage of fluorescent punctate-positive cells (\%)=number of fluorescent punctate-positive cells/number of total cells $\times 100 \%$ (at least 500 cells were counted under a fluorescence microscope). (C) The protein level of Beclin 1 of treated cells was detected using Western blot assay. y-Tubulin was used as an equal loading control. The bands were quantified by densitometric analysis. The values for Beclin 1 were corrected relative to they-tubulin and shown in histogram. All statistical significance was determined by Student's $t$-test. Columns, mean of three independent experiments; Mean $\pm \mathrm{SD} ;{ }^{\mathrm{c}} P<0.01$.

decrease in SIRT1 nuclear protein levels accompanied by an increase in intracellular ROS generation while NAC com- pletely reverses this decrease. This observation suggests that SIRT1 activity is negatively regulated by intracellular 

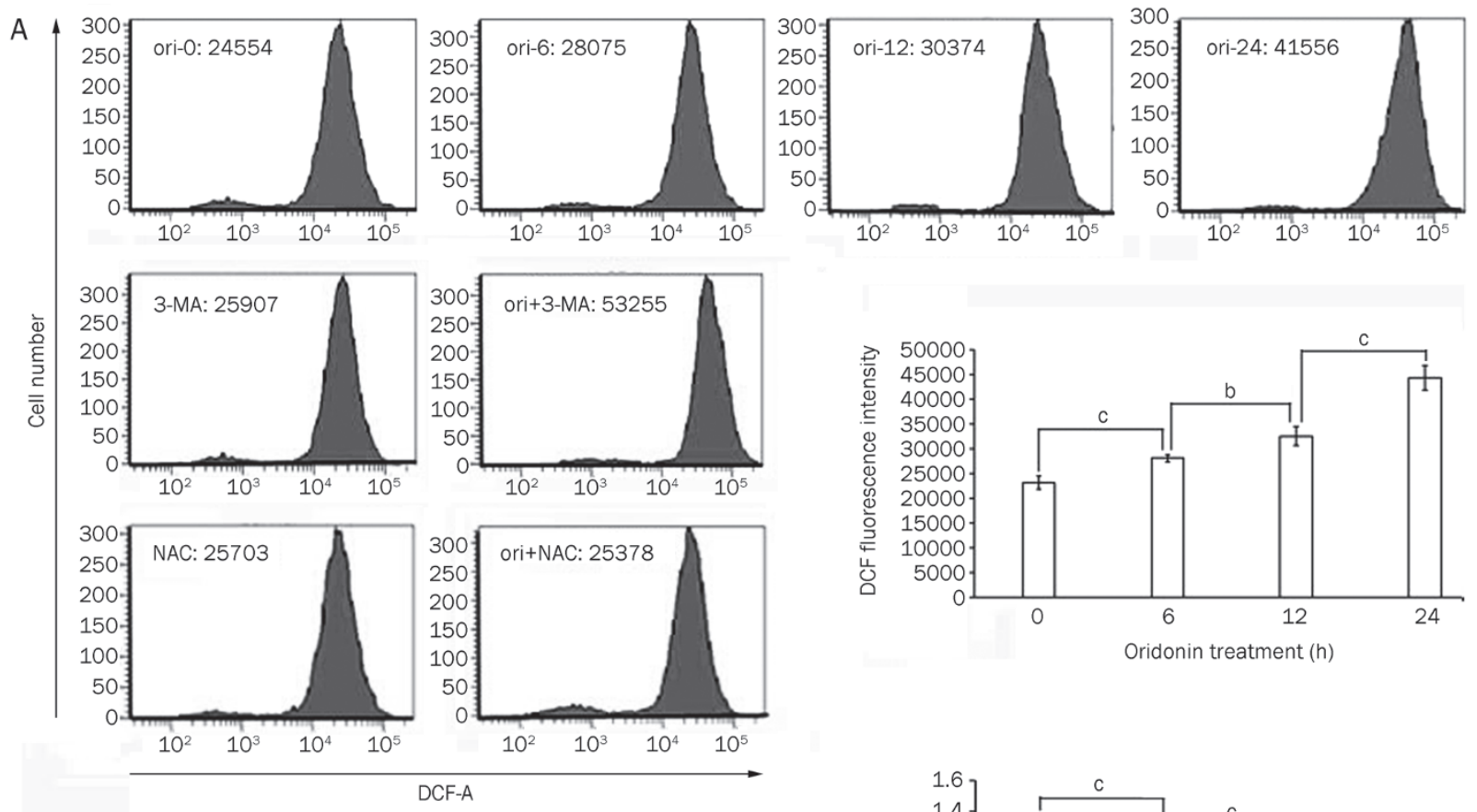

B
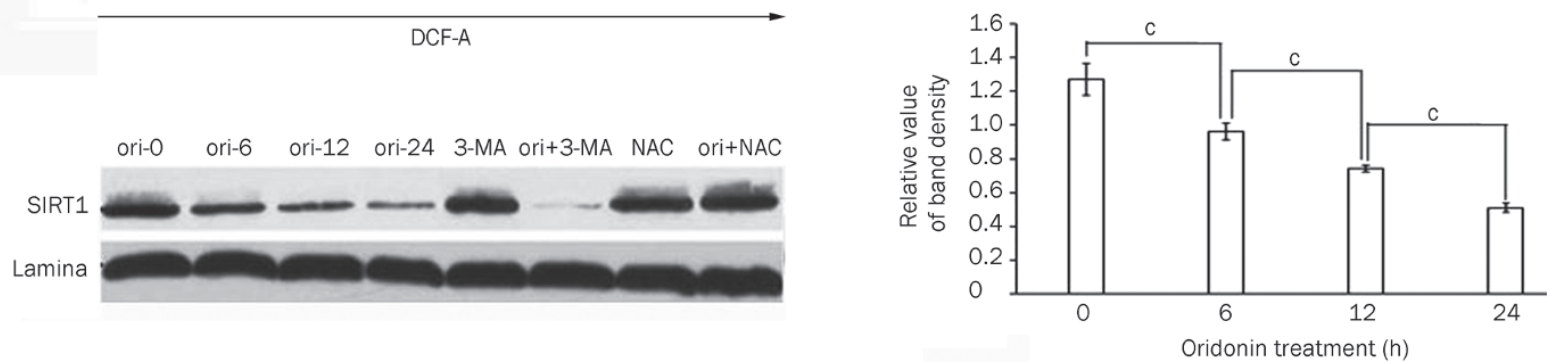

Figure 5. Assessment of the level of intracellular ROS generation and SIRT1 nucleic protein in cells treated with oridonin (ori) alone, cells preincubated with NAC and cells pre-incubated with 3-MA. The cells were treated with $7 \mu \mathrm{mol} / \mathrm{L}$ oridonin for $0,6,12$, and $24 \mathrm{~h}$, or treated with $7 \mu \mathrm{mol} / \mathrm{L}$ oridonin in the presence or absence of $5 \mathrm{mmol} / \mathrm{L} \mathrm{NAC}$, or treated with $7 \mu \mathrm{mol} / \mathrm{L}$ oridonin in the presence or absence of $5 \mathrm{mmol} / \mathrm{L} 3-\mathrm{MA}$ for $24 \mathrm{~h}$. (A) DCF fluorescent intensity was assessed by FCM analysis and shown in the histogram. (B) The levels of SIRT1 protein in nuclei were determined using Western blot assay. Nuclear lamina was used as an equal loading control. The bands were quantified by densitometric analysis and shown as histogram. All statistical significance was determined by Student's $t$-test. Columns, mean of three independent experiments; Mean $\pm S D$; ${ }^{\circ} P<0.01$.

ROS generation in RPMI8226 cells exposed to oridonin. The anti-apoptotic function of SIRT1 was self-explanatory ${ }^{[20,21,32]}$. Accordingly, we demonstrated that oridonin decreases SIRT1 nuclear protein levels and increased apoptosis whereas 3-MA enhances oridonin-induced apoptosis and the decrease in SIRT1 nuclear protein levels. However, the role of SIRT1 in autophagy is controversial. Some studies have shown that SIRT1 promotes autophagy by down-regulating mTOR signaling $^{[23]}$; however, other studies have shown that the inhibition of SIRT1 activity augmented autophagy ${ }^{[22]}$. In this study, we found that the increase in autophagy was associated with a decrease in SIRT1 nuclear protein levels and that the inhibition of autophagy by 3-MA further reduces SIRT1 nuclear protein levels and increases intracellular ROS generation. This result suggests that autophagy is negatively regulated by SIRT1 activity.

In summary, generation of intracellular ROS mediates apoptosis and autophagy in RPMI8226 cells exposed to oridonin and may be associated with a negative regulation of SIRT1 activity. Apoptosis, but not autophagy, was the major effector of oridonin-induced cytotoxicity. Autophagy protects against apoptosis mediated by intracellular ROS generation and SIRT1 activity. Our results provide new mechanistic information related to oridonin-induced apoptosis and autophagy, in addition to new MM therapeutic targets to enhance oridonin cytotoxicity.

\section{Acknowledgements}

This work was supported by grants from the National Natural Science Foundation of China (No 30871036 and 81070429).

\section{Author contribution}

Yan CHEN, Rong ZENG, and Guo-hui CUI designed research; Rong ZENG and Shuai ZHAO performed research; and Rong ZENG analyzed data and wrote the paper.

\section{References}

1 Ikezoe T, Chen SS, Tong XJ, Heber D, Taguchi H, Koeffler HP. Oridonin 

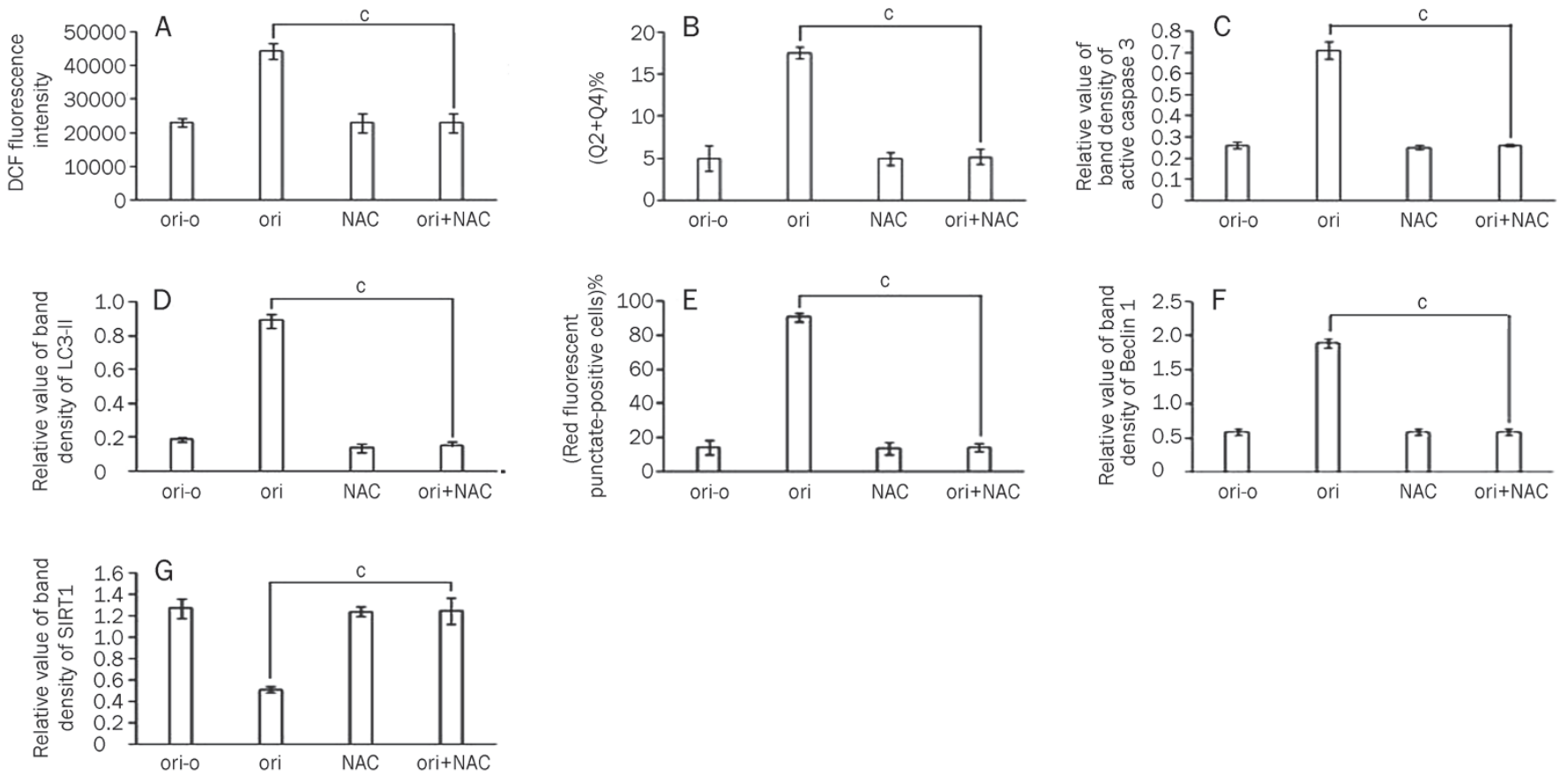

Figure 6. The effect of NAC on intracellular ROS generation, apoptosis, autophagy and SIRT1 activity in cells exposed to oridonin (ori). The data from the cells treated with $7 \mu \mathrm{mol} / \mathrm{L}$ oridonin in the presence and absence of $5 \mathrm{mmol} / \mathrm{L}$ NAC for $24 \mathrm{~h}$ were analyzed and compared with each other. Also data from the cells treated with $5 \mathrm{mmol} / \mathrm{L} \mathrm{NAC}$ for $24 \mathrm{~h}$ and cells treated in the normal media were analyzed and compared. The histograms demonstrated the data mentioned above. (A) The DCF fluorescent intensity; (B) The apoptotic rate [(Q2+Q4)\%]; (C) The protein levels of active caspase3; (D) The LC3II/Y-tubulin ratio; (E) The percentage of red fluorescent punctate-positive cells; (F) The protein levels of Beclin 1; and (G) The SIRT1 nucleic protein levels. All statistical significance was determined by Student's $t$-test. Columns, mean of three independent experiments; Mean \pm SD; ${ }^{c} P<0.01$.

induces growth inhibition and apoptosis of a variety of human cancer cells. Int J Oncol 2003; 23: 1187-93.

2 Osawa K, Yasuda H, Maruyama T, Morita H, Takeya K, Itokawa H. Antibacterial trichorabdal diterpenes from Rabdosia trichocarpa. Phytochemistry 1994; 36: 1287-91.

3 Han QB, Li ML, Li SH, Mou YK, Lin ZW, Sun HD. Ent-kaurane diterpenoids from Isodon rubescens var lushanensis. Chem Pharm Bull (Tokyo) 2003; 51: 790-3.

4 Bursch W, Ellinger A, Gerner C, Frohwein U, Schulte-Hermann R. Programmed cell death (PCD). Apoptosis, autophagic PCD, or others? Ann N Y Acad Sci 2000; 926: 1-12.

5 Shintani T, Klionsky DJ. Autophagy in health and disease: a doubleedged sword. Science 2004; 306: 990-5.

6 Tsujimoto Y, Shimizu S. Another way to die: autophagic programmed cell death. Cell Death Differ 2005; 12: 1528-34.

7 Yang ZJ, Chee CE, Huang S, Sinicrope FA. The role of autophagy in cancer: therapeutic implications. Mol Cancer Ther 2011; 10: 153341.

8 Cui Q, Tashiro S, Onodera S, Minami M, Ikejima T. Oridonin induced autophagy in human cervical carcinoma HeLa cells through Ras, JNK, and P38 regulation. J Pharmacol Sci 2007; 105: 317-25.

9 Li D, Cui Q, Chen SG, Wu L, Tashiro S, Onodera S, et al. Inactivation of ras and changes of mitochondrial membrane potential contribute to oridonin-induced autophagy in a431 cells. J Pharmacol Sci 2007; 105: 22-33.

10 Cheng Y, Qiu F, Huang J, Tashiro S, Onodera S, Ikejima T. Apoptosissuppressing and autophagy-promoting effects of calpain on oridonininduced L929 cell death. Arch Biochem Biophys 2008; 475: 148-55.
11 Hoang B, Benavides A, Shi Y, Frost P, Lichtenstein A. Effect of autophagy on multiple myeloma cell viability. Mol Cancer Ther 2009; 8: 1974-84.

12 Hou DX, Tong X, Terahara N, Luo D, Fujii M. Delphinidin 3-sambubioside, a Hibiscus anthocyanin, induces apoptosis in human leukemia cells through reactive oxygen species-mediated mitochondrial pathway. Arch Biochem Biophys 2005; 440: 101-9.

13 Chipuk JE, Bouchier-Hayes L, Green DR. Mitochondrial outer membrane permeabilization during apoptosis: the innocent bystander scenario. Cell Death Differ 2006; 13: 1396-402.

14 Lisanti MP, Martinez-Outschoorn UE, Chiavarina B, Pavlides S, Whitaker-Menezes D, Tsirigos A, et al. Understanding the "lethal" drivers of tumor-stroma co-evolution: emerging role(s) for hypoxia, oxidative stress and autophagy/mitophagy in the tumor micro-environment. Cancer Biol Ther 2010; 10: 537-42.

15 Essick EE, Sam F. Oxidative stress and autophagy in cardiac disease, neurological disorders, aging and cancer. Oxid Med Cell Longev 2010; 3: $168-77$.

16 Scherz-Shouval R, Elazar Z. Regulation of autophagy by ROS: physiology and pathology. Trends Biochem Sci 2011; 36: 30-8.

17 Grimsrud PA, Xie H, Griffin TJ, Bernlohr DA. Oxidative stress and covalent modification of protein with bioactive aldehydes. J Biol Chem 2008; 283: 21837-41.

18 Hasegawa K, Wakino S, Yoshioka K, Tatematsu S, Hara Y, Minakuchi $\mathrm{H}$, et al. Sirt1 protects against oxidative stress-induced renal tubular cell apoptosis by the bidirectional regulation of catalase expression. Biochem Biophys Res Commun 2008; 372: 51-6.

19 Caito S, Hwang JW, Chung S, Yao H, Sundar IK, Rahman I. PARP-1 

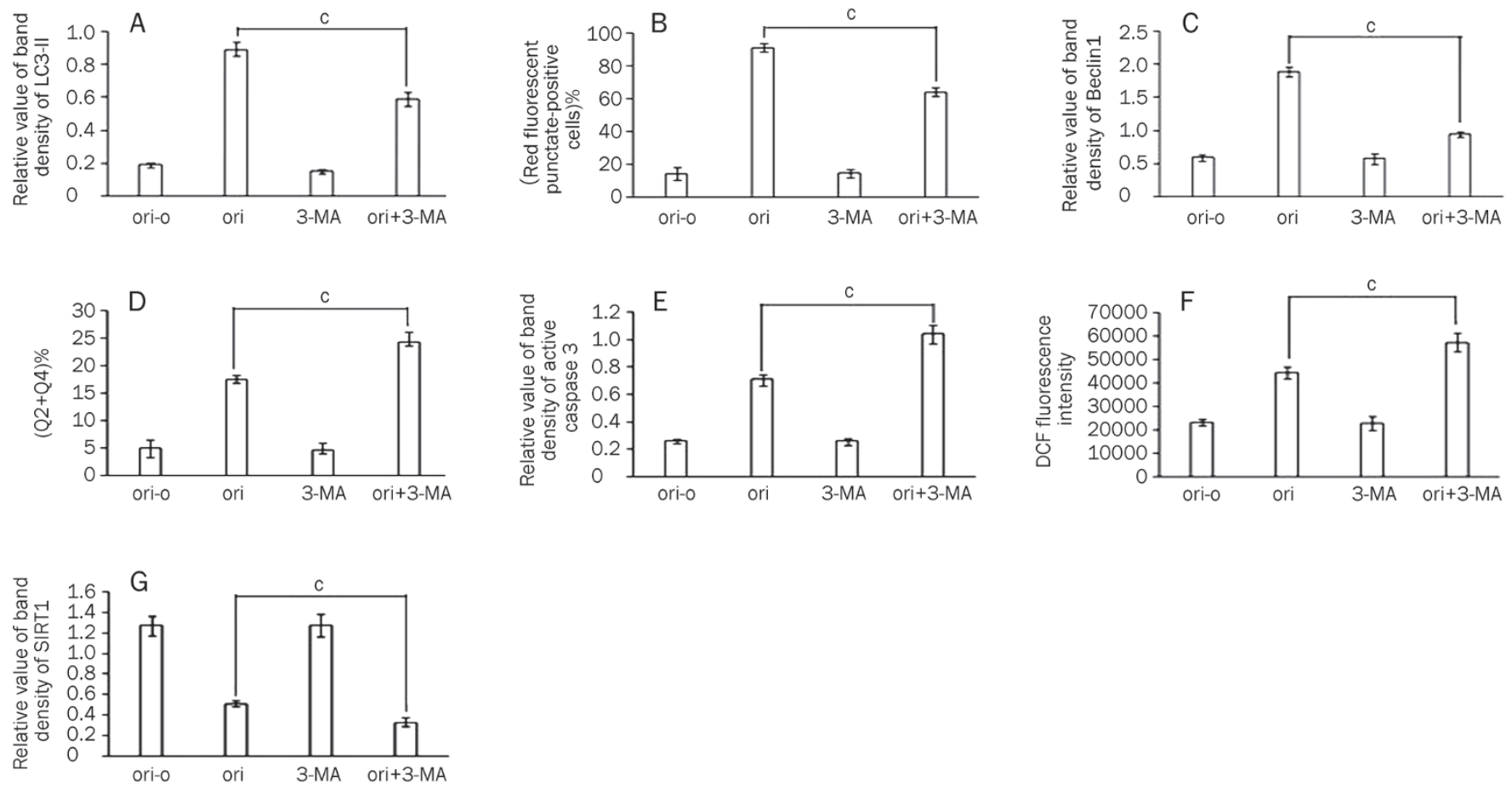

Figure 7. The effect of 3-MA on apoptosis, autophagy, intracellular ROS generation and SIRT1 activity in cells exposed to oridonin (ori). The data from the cells treated with $7 \mu \mathrm{mol} / \mathrm{L}$ oridonin in the presence and absence of $5 \mathrm{mmol} / \mathrm{L} 3-\mathrm{MA}$ for $24 \mathrm{~h}$ were analyzed and compared with each other. Also data from the cells treated with $5 \mathrm{mmol} / \mathrm{L} 3-\mathrm{MA}$ for $24 \mathrm{~h}$ and cells treated in the normal media were analyzed and compared. The histograms demonstrated the data mentioned above. (A) The LC3-II/Y-tubulin ratio; (B) The percentage of red fluorescent punctate-positive cells; (C) The protein levels of Beclin 1; (D) The apoptotic rate [(Q2+Q4)\%]; (E) The protein levels of active caspase 3; (F) The DCF fluorescent intensity; and (G) The SIRT1 nucleic protein levels. All statistical significance was determined by Student's $t$-test. Columns, mean of three independent experiments; Mean \pm SD; ${ }^{\mathrm{c}} P<0.01$.

inhibition does not restore oxidant-mediated reduction in SIRT1 activity. Biochem Biophys Res Commun 2010; 392: 264-70.

20 Ford J, Jiang M, Milner J. Cancer-specific functions of SIRT1 enable human epithelial cancer cell growth and survival. Cancer Res 2005; 65: 10457-63.

21 Kojima K, Ohhashi R, Fujita Y, Hamada N, Akao Y, Nozawa Y, et al. A role for SIRT1 in cell growth and chemoresistance in prostate cancer PC3 and DU145 cells. Biochem Biophys Res Commun 2008; 373: 423-8.

22 Hwang JW, Chung S, Sundar IK, Yao H, Arunachalam G, McBurney MW, et al. Cigarette smoke-induced autophagy is regulated by SIRT1PARP-1-dependent mechanism: implication in pathogenesis of COPD. Arch Biochem Biophys 2010; 500: 203-9.

23 Chung S, Yao H, Caito S, Hwang JW, Arunachalam G, Rahman I. Regulation of SIRT1 in cellular functions: role of polyphenols. Arch Biochem Biophys 2010; 501: 79-90.

24 Xie HY, Liang JG, Liu Y, Zhang ZL, Pang DW, He ZK, et al. Preparation and characterization of overcoated II-VI quantum dots. J Nanosci Nanotechnol 2005; 5: 880-6.

25 Kabeya Y, Mizushima N, Ueno T, Yamamoto A, Kirisako T, Noda T, et al. LC3, a mammalian homologue of yeast Apg8p, is localized in autophagosome membranes after processing. EMBO J 2000; 19: 5720-8.

26 Cui Q, Tashiro S, Onodera S, Ikejima T. Augmentation of oridonin- induced apoptosis observed with reduced autophagy. J Pharmacol Sci 2006; 101: 230-9.

27 Jia L, Dourmashkin RR, Allen PD, Gray AB, Newland AC, Kelsey SM. Inhibition of autophagy abrogates tumour necrosis factor alpha induced apoptosis in human T-lymphoblastic leukaemic cells. $\mathrm{Br} J$ Haematol 1997; 98: 673-85.

28 Zhu JH, Horbinski C, Guo F, Watkins S, Uchiyama Y, Chu CT. Regulation of autophagy by extracellular signal-regulated protein kinases during 1-methyl-4-phenylpyridinium-induced cell death. Am J Pathol 2007; 170: 75-86.

29 Chen Y, McMillan-Ward E, Kong J, Israels SJ, Gibson SB. Oxidative stress induces autophagic cell death independent of apoptosis in transformed and cancer cells. Cell Death Differ 2008; 15: 171-82.

30 Yang SR, Wright J, Bauter M, Seweryniak K, Kode A, Rahman I. Sirtuin regulates cigarette smoke-induced proinflammatory mediator release via RelA/p65 NF-kappaB in macrophages in vitro and in rat lungs in vivo: implications for chronic inflammation and aging. Am J Physiol Lung Cell Mol Physiol 2007; 292: L567-76.

31 Rajendrasozhan S, Yang SR, Kinnula VL, Rahman I. SIRT1, an antiinflammatory and antiaging protein, is decreased in lungs of patients with chronic obstructive pulmonary disease. Am J Respir Crit Care Med 2008; 177: 861-70.

32 Kim EJ, Um SJ. SIRT1: roles in aging and cancer. BMB Rep 2008; 41: 751-6. 